\title{
Criminalización y concentración de la pobreza urbana en barrios segregados. Síntomas de guetización en La Pintana, Santiago de Chile
}

Tamara Ortega $U$. Universidad de Chile, Santiago, Chile

RESUMEN | En este trabajo compartimos los resultados y conclusiones más atrayentes de una investigación mayor, la cual analizó interpretaciones y vivencias del que podría ser uno de los grupos más afectados con la fragmentación y segmentación de las ciudades actuales: los jóvenes. En un contexto de altas tasas de criminalidad y temor de la población, los procesos socializadores de jóvenes al interior de sus barrios presentan múltiples características, tanto evidentes como subterráneas. La investigación se sustenta en un enfoque de carácter cualitativo, complementado con técnicas de información geográfica -como mapas perceptuales- y entrevistas, todas ellas aproximaciones que permiten establecer tipologías de espacios socializadores. Las distintas etapas de desarrollo juvenil, los mecanismos de transmisión de conductas y la ocupación violenta del espacio público, dan cuenta de modos alternativos de reproducción de ciertos elementos subculturales. Su estudio implica intervenir en lo urbano y la juventud, sobre todo develando patrones de conformación de la ciudad que tienden a guetificar ciertos enclaves de pobreza y criminalidad.

PALABRAS CLAVE | pobreza, segregación, violencia.

ABSTRACT | In this paper, we share the most compelling findings and conclusions of a broader research project, that analyzed interpretations and experiences of what could be one of the groups most affected by the fragmentation and segmentation of the modern city: the young. In a context of high crime rates and fear, the socializing processes of young people within their neighborhoods display multiple characteristics, some of them patent, others implicit. This study uses a qualitative approach, supplemented by geo-information technologies - such as perceptual maps - and interviews, which disclose different types of socializing spaces. Different stages of youth development, the transmission of behaviors mechanisms and the violent occupation of public space, show us alternative ways in which subcultural elements are reproduced. To study them means to intervene into the urban sphere and youth, especially revealing patterns of city conformation that tend to ghettify enclaves of poverty and crime.

KEY WORDS | poverty, segregation, violence.

Recibido el 24 de enero de 2012, aprobado el 10 de septiembre de 2012

E-Mail: tamara.ortega.u@gmail.com

En este artículo se sintetizan los principales resultados de la investigación "Criminalización y concentración de la pobreza y la violencia urbana en barrios segregados: Pautas de socialización de jóvenes de La Pintana, Santiago de Chile”. Con este estudio, la autora obtuvo el título de Socióloga y el grado de Licenciada en Sociología en la Universidad de Valparaíso. 


\section{Introducción}

Entre los estudios de sociología urbana, se aprecia un interés creciente por desarrollar análisis de los procesos experimentados por jóvenes pobres de la ciudad, los cuales, además de protagonizar altos índices de desempleo, deserción escolar y precariedad, deben lidiar en forma habitual con las características más nocivas presentes en barrios segregados. Entre ellas, manifestaciones de violencia y hechos ilícitos que contribuyen a trazar el esquema de percepción y sentidos de pertenencia de la etapa adolescente.

En el contexto chileno actual, el tema de la criminalidad constituye un problema abordado desde múltiples ámbitos, incluso desde la opinión pública y en forma mediática. Sin embargo, existen escasos análisis que intenten comprender el problema desde los procesos socializadores que acompañan a los segmentos juveniles en situación de pobreza y exclusión. En un intento de suplir ese vacío, el artículo indaga en las dimensiones que alcanzan estos procesos socializadores en contextos de exclusión social y confinamiento espacial, y su expresión en las conductas de grupos determinados de la población. A tal fin se analizan los ejes que articulan formas de vida atravesadas por el nexo entre barrio, socialización y violencia juvenil, en un ámbito local específicos.

Resulta interesante otorgar relevancia al estudio de aquellos sujetos que cometen delitos menores y que se encuentran en situación inaugural de comportamiento delictivo. Con ello aportamos a la generación de conocimiento acerca del aprendizaje de conductas delictivas en jóvenes de corta edad, relevando el rol fundamental de las políticas públicas locales para la superación de este problema social, visto por lo general desde el piso central de exclusión, pobreza y violencia juvenil que envuelve a aquellos comportamientos.

La intervención de la sociología urbana resulta crucial en el esfuerzo por recuperar la interacción e integración entre los grupos sociales, incluyendo un espacio público que permita renovar las certidumbres y confianzas básicas entre las personas, así como obtener un sentimiento de pertenencia hacia un tejido social activo y más participativo.

El artículo presenta antecedentes contextuales, teóricos, método y resultados principales, otorgando mayor énfasis a las conclusiones obtenidas en torno a aspectos interpretativos y definición de tipologías espaciales ${ }^{1}$.

\section{Antecedentes de contexto}

\section{Procesos de globalización y urbanización}

En la actualidad, gran parte de las ciudades latinoamericanas participa activamente en los procesos de reestructuración capitalista, caracterizados por cambios en la informatización y flexibilización de los mercados, en las nuevas formas de tecnología y comunicación, además de transformaciones en la dinámica productiva. Estos

1 Para revisar su versión completa, la cual posee un extenso análisis cualitativo sobre interpretaciones juveniles, se debe acudir a la autora. 
procesos forman parte de la tendencia globalizadora presente en la región - de la cual Chile participa activamente-, con su secuela de transformaciones que han llevado a las ciudades latinoamericanas a constituirse en máquinas de crecimiento urbano capitalista. Uno de los resultados de este desarrollo ha sido la generación de nuevas formas de configuración espacial caracterizadas, entre otras cosas, por fragmentación social, polarización y segregación residencial (Ducci, 2004).

Por su parte, el actual rol del Estado es de desregulación frente a variadas situaciones de la realidad social, entre las que se encuentra la gestión urbana, que actualmente le otorga al capital privado un papel central como promotor del desarrollo urbano. En este sentido, desde los ańos ochenta la ciudad de Santiago presenta una serie de transformaciones en su configuración espacial, que han tenido lugar - principalmente desde el gobierno dictatorial- a través de un poderoso mecanismo: la erradicación forzada de 'campamentos'. Esta intervención desencadenó profundas rupturas en las comunidades, complejos procesos de reestructuración, marginalidad y concentración espacial de la pobreza, que generaron nuevas problemáticas en aquellos sectores menos favorecidos.

Según algunos autores, Santiago corresponde a "una ciudad segregada en términos socioeconómicos, con áreas urbanas fragmentadas desde el punto de vista administrativo, con inequidades en la calidad de los servicios públicos, y creciente inquietud, temor y percepción de inseguridad en la población" (Rodríguez \& Winchester, 2001, p. 18). Además, las políticas públicas habitacionales han desarrollado una organización segmentada y segregada de las viviendas, acorde a los estratos socioeconómicos de la población (Rodríguez \& Sugranyes, 2004; Hidalgo, 2007; Rodríguez, 2005; Ducci, 2004; De Mattos, 2004; Kaztman, 2001), interviniendo también los precios del suelo en la construcción de esta trama socioespacial (Sabatini, 2000; Arriagada \& Simioni, 2000).

Por otra parte, a pesar de que la pobreza ha disminuido en términos generales, los patrones de distribución espacial de la pobreza siguen siendo similares a los de décadas anteriores. El área urbana de Santiago está claramente diferenciada según los niveles de ingreso de las familias, lo que se refleja en importantes desniveles en la calidad y volumen de infraestructura y equipamiento ${ }^{2}$. A la par de estas tendencias se generan barrios segregados al interior de la ciudad que reproducen las desigualdades socioeconómicas, puesto que, al ser excluidos, los pobres mantienen un contexto de pobreza y pares pobres, lo que reduce su margen de posibilidades, sus contactos y sus probabilidades de exposición a códigos, mensajes y conductas funcionales a una movilidad social ascendente.

En el marco descrito sucintamente, y como consecuencia de él, la ciudad de Santiago se caracteriza por dos principales rasgos de su organización espacial. En primer lugar, un desarrollo desigual donde coexisten, por una parte, una población cosmopolita, globalizada, con empleos estables y salarios altos, mientras que,

2 Un análisis de la pobreza e indigencia según municipalidades en Santiago muestra que, en nueve comunas, la pobreza se encuentra entre el 0 y $10 \%$ de la población; en once comunas, entre 10 y $20 \%$; y solo en cinco comunas se concentra entre el 20 y $30 \%$. Los extremos fluctúan entre 1,6\% de pobreza en Nunnoa, una comuna habitada casi en su totalidad por sectores de ingresos medios, y el 29,2\% en La Pintana (Rodríguez \& Winchester, 2001). 
por otra, se encuentra una población pobre, desempleada, marginal y criminalizada (De Mattos, 1999, 2002). En segundo lugar, la existencia de guetos de pobreza y exclusión del "sistema globalizado", en los cuales se manifiestan formas alternativas de convivencia y socialización propias de un segmento apartado y aislado, en términos espaciales y culturales, donde se asimilan ciertos patrones como cotidianos y normales, y cuyos actores aparecen ante el resto de la población como socialmente desviados. Estos barrios suelen caracterizarse por presentar altos niveles de exclusión social, vulnerabilidad y estigmatización (Kaztman, 2001 y Katzman et al., 2003; Lunecke, 2004; Sabatini, 1999; Saraví, 2004), además de presentar altos niveles de victimización y temor en la población y una menor presencia legal del Estado (Ministerio del Interior, Chile, 2004; Dammert \& Oviedo, 2004; Frühling \& Sandoval, 1997).

\section{La delincuencia juvenil}

El fenómeno de la delincuencia se ha constituido en uno de los temas relevantes en la agenda pública, privada y académica. El aumento de la delincuencia ha provocado un gran sentimiento de inseguridad en la población, reflejado en la importancia que le atribuyen los chilenos. Así, según el Programa de Naciones Unidas para el Desarrollo (PNUD, 1998), "los chilenos suelen asociar espontáneamente la inseguridad con la delincuencia”.

Es preciso señalar que la relación causal entre pobreza y delincuencia es rechazada por varios autores (Arriagada \& Godoy, 2000; Tironi, Weinstein \& Martínez, 1990 , entre otros). Se ha indicado, en cambio, otro elemento fundamental que interviene mayormente en la criminalidad: la inequidad (Dammert, 2005), rasgo prevalente en nuestro país donde el crecimiento en economía no ha ido acompañado de una distribución equitativa del bienestar hacia toda la población ${ }^{3}$. Se trata de una realidad de la cual es necesario hacerse cargo desde diferentes perspectivas, entre las cuales una es intentar romper el estigma analizando los factores que intervienen en la asociación entre pobreza y delincuencia.

En este contexto, últimamente a los jóvenes "les ha afectado una mayor tasa de desempleo sectorial; una mayor presión competitiva; una altísima represión 'por sospecha'; un menor acceso a la educación superior; una decreciente proyección a identidades colectivas; una menor pertenencia a familias integradas; un grado descendente de politización; una curva ascendente de actitudes anómicas, escapistas, etc." (Tijoux, 1995, p. 7). Además, existen estudios que confirman la relevancia que adquiere el espacio barrial en la socialización juvenil y en las probabilidades de ser víctima de hechos delictivos o de participar en ellos (Arriagada \& Rodríguez, 2004), sobre todo en edades tempranas.

Cabe señalar que si bien no es lo mismo ser "pobre" que ser "delincuente", y que precisamente su asociación es parte del estigma, las comunas con más altos índices de pobreza también presentan mayores porcentajes de población penal. Así ocurre con La Pintana, ya que esta comuna se encuentra en segundo lugar a nivel nacional y en primer lugar a nivel regional en cuanto a la población penal originaria de la comuna (Fernández, 2004). 


\section{Antecedentes teóricos}

Mediante una rápida revisión de enfoques teóricos atinentes al estudio, se exponen los principales argumentos que dan soporte conceptual tanto al enfoque de la problemática investigada como a los resultados obtenidos en los hallazgos empíricos. Tal revisión se realizará a partir de cuatro ejes teóricos: pobreza y exclusión, efecto barrio, socialización y delincuencia, 'lugarización' y gueto.

\section{Pobreza urbana y exclusión social}

Producto de las transformaciones económicas, sociales y culturales de las últimas décadas existe un nuevo tipo de pobreza urbana, caracterizada no por carecer de elementos materiales, como la pobreza de otrora, sino que en términos de la merma en el acceso a servicios básicos por parte de diversos segmentos sociales, situación que afecta su calidad de vida. La lógica segregadora y el factor espacial se tornan centrales en el análisis de esta nueva pobreza urbana, así como las complejas consecuencias sociales y culturales que provoca la concentración de población en condiciones de precariedad social, más que material.

Entre las principales características de esta nueva pobreza se encuentran los procesos de pauperización de la ciudad y la exclusión de vastos sectores de la población en cuanto al acceso a bienes, servicios y oportunidades de distinto tipo, situación que deteriora el tejido social al interior de las comunidades afectadas (Kaztman, 2001; Katzman et al., 2003). Esta segregación de grupos sociales socava, además, la creación de capital cultural y participación social entre los vecinos, a la vez que desintegra y fragmenta las relaciones sociales (Cariola, 2002), contribuyendo incluso a la formación de guetos urbanos (Katzman et al., 2003; Wacquant, 2001). La ciudad se torna un factor preponderante a la hora de influir en la capacidad de inclusión o exclusión de los procesos y sistemas sociales.

El concepto de exclusión sugiere que ciertos individuos permanecen omitidos de los procesos de desarrollo, como consecuencia de una débil capacidad de integración por parte del modelo social. Existen procesos que determinan la exclusión, procesos económicos, políticos y culturales (Balbo, Jordán \& Simioni, 2003). En el caso de La Pintana, resulta paradigmático el análisis de vivencias particulares, puesto que se manifiesta la exclusión social a través de las tres variantes mencionadas por el estudio de Balbo, Jordán y Simioni: a) exclusión de bienes y servicios urbanos básicos; b) exclusión de un trabajo estable y remunerado regularmente; y c) exclusión de participación en la toma de decisiones políticas.

Sumado a lo anterior, el contraste en los ingresos y las desigualdades en la estabilidad laboral también se ven reflejados en la localización diferenciada de las clases sociales en el territorio urbano, reduciéndose las áreas de interacción entre ellas. Tal aspecto genera un creciente aislamiento social de los pobres urbanos respecto de las "corrientes principales de la sociedad", lo que se convierte en un obstáculo para acumular los activos necesarios para una movilidad social ascendente (Kaztman, 2001).

En definitiva, la conceptualización de pobreza urbana, en este caso, posee un fuerte vínculo con la exclusión social y segregación espacial, con un énfasis especial en la ubicación de los grupos sociales en las grandes ciudades y la posibilidad o no 
de reunirse, interactuar y articular patrones de conducta que no generen aislamiento de ciertos grupos, de sus códigos, valores y comportamientos.

\section{Efecto barrio, socialización y delincuencia}

Existen investigaciones en torno al 'efecto barrio', entendiéndolo como las distintas maneras en que la concentración espacial de la pobreza moldea ciertos mecanismos de socialización barriales (Flores, 2006). De esta forma, las decisiones de los individuos se encuentran afectadas por las características contextuales del vecindario. Estos estudios indagan en las dimensiones y efectos provocados por la segregación residencial socioeconómica y las características de los grupos afectados al enfrentarse a los mecanismos institucionales de socialización, como la familia y la escuela. Además, señalan el vacío existente en el estudio de estos fenómenos desde la perspectiva del contexto barrial.

En este enfoque, el efecto provocado por el barrio en un entorno de pobreza, exclusión y segregación intervendría en la generación o ausencia de oportunidades, estímulos o imágenes positivas de superación y movilidad social, donde el capital social existente no estimula ni permite dejar la pobreza recurriendo a los parámetros aceptados por la sociedad (Kaztman, 2001). No obstante, se resalta la importancia que adquiere la capacidad de los individuos de resistirse a la realidad barrial y anular el efecto barrio negativo (Flores, 2006), asumiendo y creando otras oportunidades que superen el contexto vulnerable en el que se desenvuelven.

Por otra parte, algunos estudios se enfocan en los procesos de aprendizaje social en los sectores con carencias homogéneas y en su relación con problemas sociales percibidos por los mismos actores sociales acerca de su barrio (Contreras \& Weason, 2006; Lunecke \& Ruiz, 2006; Rodríguez \& Sugranyes, 2004). De acuerdo con estos autores, la situación de los barrios más carenciados se ve agravada por las características desplegadas por los jóvenes pertenecientes a dichos entornos en los procesos de aprendizaje social y la socialización entre pares, a raíz de que constituyen un grupo "especialmente sensible a las modalidades de concentración territorial de la pobreza” (Arriagada \& Rodríguez, 2004, p. 19). En esto radica la importancia de la concentración territorial de estos grupos, puesto que resulta efectiva la posibilidad de socializar conductas delictivas para los jóvenes, los que tienden a compartir con sus pares similares elementos de aprendizaje familiar y barrial, cargado de quiebres emocionales y entornos de violencia.

Respecto de la criminalidad, básicamente su teorización se basa en establecer la acción delictiva como una disfunción o 'patología social' que impide el correcto funcionamiento y equilibrio del sistema social. En la presente investigación se considera la existencia de subculturas al interior de los barrios segregados, y especialmente entre los jóvenes; por lo tanto, se asume la existencia de códigos y valores propios que se alejan de las predominantes a nivel social, pero que no dejan de superponerse en algunos ámbitos y de asimilar normas o prácticas similares, pero con distintos significados (Cohen, 1955, en García-Pablos, 1994; Cooper, 2002).

$\mathrm{Al}$ observar las políticas destinadas a jóvenes infractores, se aprecia que tienden a limitar su acción a factores protectores dentro de estos mismos entornos, más que a considerar elementos internos del contexto vital de cada sujeto (Mettifogo \& 
Sepúlveda, 2005, p. 11) y de sus pares. Es necesario, entonces, ampliar las entradas para investigar el tema juvenil, haciéndolo no solamente desde los ámbitos conservadores relacionados con la educación, el trabajo, la familia o la sexualidad, sino incorporando la problemática juvenil de infracción a la ley y factores de riesgo asociados a su socialización (Mettifogo \& Sepúlveda, 2005, p. 15).

\section{'Lugarización' y gueto}

Respecto de los significados que adquiere el entorno en etapas de socialización de

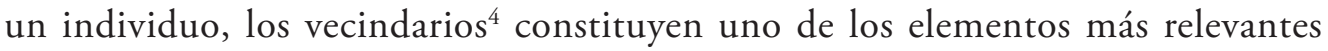
en la construcción de la personalidad, de los valores y trayectorias vitales de los grupos sociales. Más aún, en etapas adolescentes se comprende que la significación que los jóvenes atribuyen a las prácticas y sentidos compartidos en sus vecindarios, complementan la labor de las instituciones socializadoras, como la familia y la escuela.

La definición de estos lugares por los jóvenes se asocia con la posibilidad que genera la 'lugarización' del espacio residencial, en el sentido de que "cuando el espacio adquiere significación se transforma en lugar, como 'tiempo en el espacio', lo que implica un proceso de 'lugarización' a partir de la experiencia y la asignación de significado" (De la Puente et al., 1993, p. 153). Es a partir de esta lugarización que se construye, en un momento determinado, la relación entre las conductas de los actores sociales y el entorno barrial donde se desenvuelven.

En efecto, la imagen del barrio se articula, en estos contextos, tanto desde (las causas) como hacia (los efectos de) la segregación residencial socioeconómica experimentada en las percepciones, disposiciones y conductas de los habitantes de estos espacios. Según Loïc Wacquant, los elementos que debe contener un fenómeno analizado para designarlo como gueto son "un pabellón urbano delimitado, una red de instituciones específicas al grupo y una constelación cultural y cognoscitiva (valores, actitud mental o mentalidad) que conllevan al aislamiento socio-moral de una categoría estigmatizada, así como el truncamiento sistemático del espacio vital y las oportunidades de vida de sus miembros" (Wacquant, 2001, p. 73).

Con base en los hallazgos empíricos del estudio, se expondrá de qué forma estos aspectos se manifiestan en los grupos de jóvenes de un barrio de la comuna de La Pintana. Si bien es cierto los objetivos del mismo no se enfocaban en descubrir la aplicación empírica de estas teorías, de acuerdo con los resultados obtenidos es posible postular que existen características de guetización en esta realidad, lo que eventualmente se encontraría en otros lugares de las grandes ciudades de Chile.

4 Si bien es cierto el concepto de 'vecindario' ha sido abordado teóricamente en forma particular e independiente de las conceptualizaciones de barrio o espacio público, para efectos de este estudio el vecindario se considera según el contexto del análisis en forma indistinta a los otros conceptos. Para consultar respecto de las teorías en torno al vecindario y barrio en forma diacrónica, véase Gravano (2005). 


\section{Enfoque de estudio y metodología}

La línea de investigación se enfoca más en comprender e interpretar las formas y mecanismos de socialización de los jóvenes que en generar factores explicativos del delito en un caso como la comuna de La Pintana. Lo fundamental es conocer sus pautas de socialización desde las formas de interacción social alternativas a las predominantes en la sociedad. No se busca descubrir aquellos códigos y valores propios de una subcultura, ni saber por qué delinquen, sino cómo se insertan en el mundo delictual desde edades tempranas, descubrir los instrumentos que ayudan a iniciar a los jóvenes en el actuar delictivo, a partir del supuesto de que el espacio público de un barrio segregado facilita esta socialización y acercamiento a las conductas violentas y delictivas.

En este marco, el análisis se efectuó a partir de una simbiosis de diferentes técnicas, con especial énfasis en la estrategia cualitativa, la técnica de mapas perceptuales y sistemas de información georreferenciada, con el software Arc View.

El lugar de estudio se seleccionó a raíz de que La Pintana representa una comuna emblemática por distintos factores: nivel de pobreza, altos índices de delincuencia, alta presencia de población penal, incursión de jóvenes en actos delictivos, estigmatización de sus habitantes, presencia recurrente en la prensa debido a la delincuencia y origen en las políticas habitacionales de erradicaciones que llevó a cabo la dictadura militar como parte de un cambio drástico en la distribución espacial de la población. Todas estas características son compartidas por otras comunas de grandes ciudades de Chile, aquellas que se ubican en sus periferias y que contienen similares patrones en términos de delincuencia, pobreza urbana y exclusión social (en empleo y educación, por ejemplo). Además, una de las características que une a diferentes sectores pobres de la ciudad de Santiago, notoria en este caso, radica en la alta presencia de jóvenes en hechos delictivos y violentos, lo cual mantiene en permanente estado de inseguridad y temor al resto de sus habitantes.

Para efectos de seleccionar el universo empírico se han acotado dos sectores geográficos de la comuna, El Roble y El Castillo. Se han seleccionado porque, pese a poseer características homogéneas en su conformación, como tamaño, antigüedad, procesos de ocupación y densidad, entre otras, suelen manifestar interesantes elementos diferenciadores. En ambas localidades, los sujetos seleccionados corresponden a jóvenes catalogados como infractores de la ley. En consecuencia, tenemos que el universo teórico de la investigación corresponde a todos los jóvenes entre 12 y 17 ańos de la comuna de La Pintana que hayan cometido algún tipo de infracción a la ley. Se ha recurrido a registros entregados por Carabineros entre septiembre de 2007 y febrero de 2008, referentes a jóvenes hombres y mujeres de los sectores de El Castillo y El Roble de La Pintana. 


\section{Resultados}

\section{Caracterización de barrios estudiados}

La comuna de La Pintana se encuentra ubicada en la periferia sur del Gran Santiago, entre las comunas de Puente Alto, La Granja, San Ramón, El Bosque, La Florida y San Bernardo, con una superficie de 3.031 hectáreas. La población total, de acuerdo con el Censo del año 2002, es de 190.085 habitantes, que representan el 4,83\% de la población de Santiago.

Es interesante observar el patrón de crecimiento de la población de la comuna entre los años 1982 y 1992, entre los cuales la población comunal aumentó en 95.708 habitantes, mientras entre 1992 y 2002 lo hizo en 25.201 personas. Es, por lo tanto, en el periodo inmediatamente posterior a la creación de la comuna (1984) en el que se registra una mayor tasa de crecimiento, en el contexto de las radicaciones y erradicaciones determinadas por las políticas urbanas del Estado.

En términos administrativos, la Municipalidad de La Pintana ha establecido una división interna de la comuna en cuatro sectores. Esto configura cuatro espacios geográficos claramente delimitados, con historias particulares que contribuyen a definir el contexto de la historia e identidad comunal. En la Figura 1 se muestra la ubicación de estos cuatro sectores, identificándose los dos sectores estudiados.

\section{Figura 1 | Sectores estudiados de La Pintana}

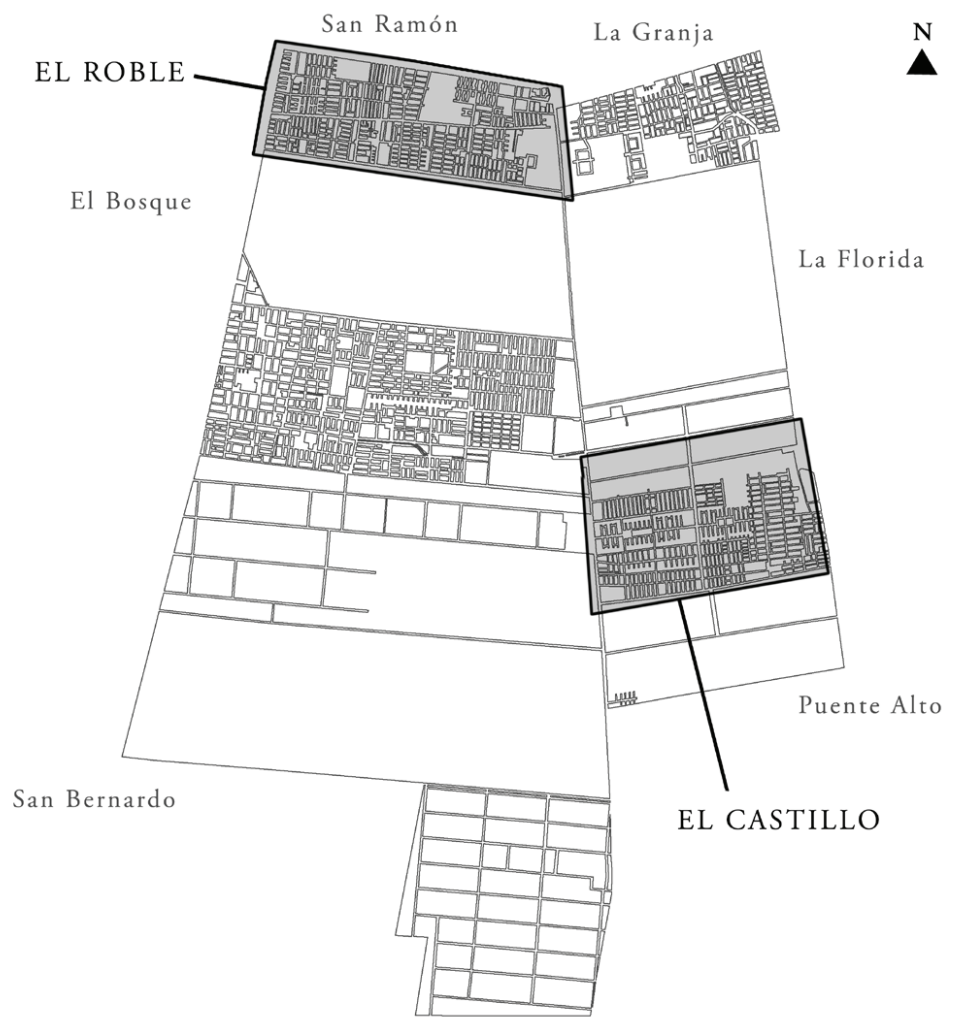

FUENTE SECRETARÍA DE PLANIFICACIÓN COMUNAL (SECPLAC), MUNICIPALIDAD DE LA PINTANA (2008) 


\section{Caracterización de los jóvenes ${ }^{5}$}

Las edades de los jóvenes fluctúan entre los 11 y 18 años de edad, siendo el promedio 15 años. En el conjunto de la lista analizada existe mayor presencia de hombres (74) que de mujeres (25), con una mayor incidencia masculina, de 74,7\%, frente al $25,2 \%$ femenina.

Respecto de las familias ${ }^{6}$, el número promedio de integrantes del grupo familiar es de 5,6 personas. El 47,4\% pertenece a familias monoparentales, el 36,3\% a biparentales, un $9 \%$ a familias extendidas y un $4 \%$ a familias reensambladas.

Por su parte, el nivel de escolarización es uno de los antecedentes de mayor preocupación en estos sectores, puesto que la mayoría de los casos se distribuye entre séptimo básico y segundo medio, con el 37\% de los jóvenes entre séptimo y octavo básico. No obstante, dado que estos datos arrojan resultados incompletos, para efectos de caracterizar el nivel educativo de esta población utilizaremos el indicador Rezago Educativo, que expresa la diferencia existente entre los ańos de estudio esperados para una edad respecto de los ańos de estudio observados ${ }^{7}$. De acuerdo con este indicador, alrededor de un cuarto de los jóvenes $(27,9 \%)$ cursa el nivel educativo esperable para su edad, con cero ańos de rezago, mientras el $72 \%$ presenta algún nivel de rezago (entre uno y diez ańos) ${ }^{8}$. Un $23,7 \%$ tiene más de cuatro ańos de rezago, lo que deja en evidencia una grave situación de irregularidad educativa asociada a la juventud con antecedentes delictuales. Particularmente preocupante es el 10,8\% que registra entre seis y diez años de retraso, los que son de muy difícil recuperación, sobre todo tomando en consideración el encadenamiento con otros factores de exclusión social presentes en estos jóvenes.

Los actos ilícitos analizados corresponden a los Delitos de Mayor Connotación Social. Al respecto, la participación de los jóvenes en los comportamientos tipificados en esa categoría arroja también conductas propias del incipiente actuar delictivo, como conducir sin licencia, comercio ambulante o desórdenes. De acuerdo con las tipificaciones de delitos que suelen cometer los jóvenes a nivel general, el Hurto es el hecho predominante entre sus conductas en conflicto con la ley, en este caso constituyendo un 65,6\% de los casos. En segundo lugar se encuentra el Robo con Intimidación, con un 11,1\%. Los delitos restantes no superan el 5\% de los sucesos reportados.

Para datos cuantitativos en gráficos y tablas se puede consultar la investigación completa con la autora. Entendiendo que las familias se clasifican de acuerdo con la composición de sus integrantes y su relación de parentesco. La familia biparental es aquella conformada por ambos padres (padre o madre); la familia monoparental está conformada por uno u otro padre (padre o madre) y los hijos; la familia reensamblada es aquella compuesta por un padre y una madre con un lazo anterior descompuesto o quebrado (de uno de ambos) y sus hijos, si los tuvieren; y por último, la familia extendida es aquella constituida por padre y/o madre, hijos y demás ascendientes, descendientes o colaterales (abuelos, tíos, primos). Una familia extendida puede estar compuesta por varias familias nucleares.

7 Por ejemplo, para un joven de 15 años se espera que, si no ha repetido ningún curso, se encuentre cursando $1^{\circ}$ medio. Si cursa $7^{\circ}$ básico, entonces tenemos para este caso un rezago educativo de dos ańos.

8 Para la Región Metropolitana tenemos un 48\% respecto de un ańo de rezago en jóvenes entre 15 y 17 ańos. Según información extraída el 22 de diciembre de 2009 en http://celade.eclac.cl/cgibin/ RpWebEngine.exe/MultiFilter 
En relación con la comuna en que fueron detenidos, los datos disponibles indican que de 41 casos, 25 fueron aprehendidos en comunas colindantes (60,9\%), como Puente Alto, San Bernardo o San Ramón; diez (24,3\%) lo fueron en comunas lejanas (centro y sector oriente), como Las Condes, Macul y Providencia, mientras que en La Pintana se detuvo a solo seis jóvenes, correspondientes al 14,6\% de los casos. Estas cifras indican que los comportamientos delictivos de estos grupos también responden a patrones de segregación de la ciudad, al reflejar la menor disposición de la juventud a acudir a otros sectores de la ciudad para realizar tales conductas, manteniéndose en este 'rincón' de Santiago.

\section{Mecanismos de socialización en espacios públicos segregados}

La transmisión de conductas ilícitas entre los jóvenes de estos barrios atraviesa múltiples aristas relacionadas con procesos de socialización sustentados en experiencias de vida y prácticas cotidianas, donde intervienen trayectorias familiares y educacionales, experiencias con sus grupos de pares y elementos del entorno barrial, entre otras. En esta oportunidad revisaremos uno de los ejes centrales de la investigación, relativo a las experiencias cognitivas y conductuales adquiridas en el espacio público barrial y que son reforzadas por el importante rol ejercido por el grupo de pares.

Entre los jóvenes, la definición física del barrio denota una percepción del entorno inmediato que logra articular su propio sentido de pertenencia. En este aspecto, la inseguridad resulta un factor decisivo en la percepción del barrio, tanto para el uso como para la apropiación simbólica del mismo. Ello obedece mayormente a las experiencias adquiridas con otros grupos de jóvenes, o bien al conocimiento o imagen del barrio que algunos sectores representan para el resto de la comunidad.

A partir de la forma de utilización y apropiación del barrio se identifican tipologías que responden a la existencia de lugares cargados de significaciones compartidas y que, además, contribuyen a establecer trayectorias y modos de uso de los mismos. $\mathrm{Al}$ respecto, el presente apartado muestra los espacios públicos en términos físicos y de significados, declarados por los jóvenes a través de la técnica de mapas perceptuales aplicados en cada entrevista.

Las tipificaciones se obtienen a través de los atributos que posee el espacio para los jóvenes, los cuales arrojan dos grandes dimensiones de análisis: a) lugares de mayor apropiación; y b) lugares que presentan dinámicas complejas en cuanto a la presencia de hechos ilícitos y que muchas veces generan atracción (o rechazo en algunos casos) para sus actividades entre los grupos de jóvenes.

Ambos tipos de significados evidencian elementos que caracterizan las expectativas, intereses y gustos de los jóvenes, vinculados a sus vecindarios, y que también aportan en la construcción de la concepción que poseen los grupos juveniles acerca del espacio público de sus lugares de residencia. En virtud de analizar las formas de lugarización presentes en los barrios estudiados, se revisan ambos sectores con sus significados en términos generales, a fin de conocer las apreciaciones juveniles en cada barrio. 
Tipologías de espacios públicos: uso, delito y lugarización

Para los jóvenes, la conformación de los barrios se encuentra atravesada por la presencia de ciertos límites al interior del sector, que contribuyen a circunscribir sus prácticas y usos del espacio, según las conductas desarrolladas en ellos. En primer lugar, se identifica la presencia de límites que dividen los sectores estudiados en subsectores con cargas simbólicas que se sustentan en la mayor o menor peligrosidad del barrio, o el predominio de conductas ilícitas al interior de los mismos. Esta situación refleja la importancia del sentido de pertenencia respecto de los distintos subsectores reconocidos por los jóvenes, puesto que, de acuerdo con esta pertenencia, los sujetos construyen su identificación con los habitantes de cada barrio, a la vez que organizan sus trayectorias dentro del mismo.

FIGURA 2 | Sector El Roble. Identificación de tipologías barriales según los jóvenes

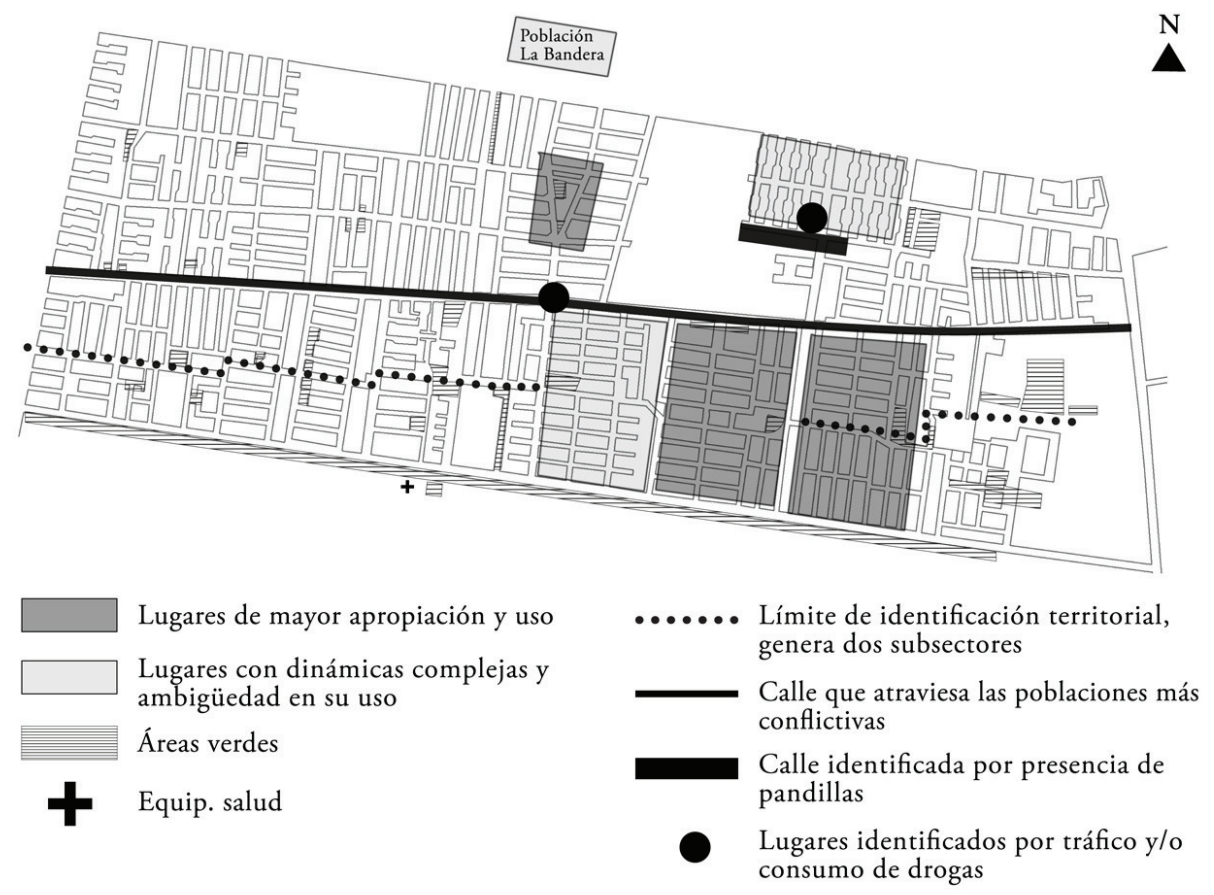

FUENTE ELABORACIÓN PROPIA A PARTIR DE INFORMACIÓN OBTENIDA DE MAPAS PERCEPTUALES

Como muestra la Figura 2, para el sector de El Roble se aprecian líneas divisorias, establecidas por las apreciaciones juveniles en torno a la cartografía utilizada en las entrevistas. En primer lugar, la avenida General Francisco Franco (simbolizada con la línea negra) divide el sector en dos segmentos, a partir de lo que los sujetos indican como 'su población'; es decir, la delimitación de pertenencia a sus propios barrios se circunscribe en función de la frontera designada por la delimitación administrativa de las poblaciones. Concretamente, a partir de una de las avenidas principales se construye la visión de pertenencia a uno u otro subsector $y$, por ende, se manifiesta la diferencia existente entre los habitantes y las conductas del otro lado del límite. En este sentido, los jóvenes señalan sentirse parte de uno u otro lado de 
la línea divisoria dependiendo de su cercanía con las prácticas desarrolladas por los habitantes de cada sitio.

En segundo lugar, a través de la línea punteada se representa una especie de conexión entre aquellas poblaciones con mayores significados conflictivos para los jóvenes (calle Antonio Machado). Esta calle es uno de los lugares donde es posible encontrar pautas de comportamiento como consumo y tráfico de drogas, encuentros entre grupos que podrían terminar en peleas callejeras, y donde el transitar generaría una especie de 'estatus' entre los grupos de jóvenes que frecuentan tales poblaciones.

Existen significados en torno a la presencia de límites naturalizados para los jóvenes, representados por avenidas que cruzan todas las poblaciones del sector y a partir de las cuales se van desarrollando las trayectorias y comportamientos de los habitantes. Por ejemplo, en la Figura 2, las poblaciones indicadas con los cuadros gris oscuro constituyen dos villas de gran atracción para los jóvenes, puesto que existe mayor uso de las plazas y pasajes de ambas, ya sea por un tránsito habitual o relacionado con fiestas que durante los fines de semana se realizan en ellos, o simplemente porque se sienten parte del espacio público existente dentro de estos límites, diferenciados de los de otras poblaciones.

Resulta significativo que precisamente las mismas poblaciones identificadas en la Figura 2 (cuadros gris oscuro) como las de mayor apropiación, sean las que presentan mayor cantidad de jóvenes en conflicto con la justicia.

Por otra parte, frente a aquellos lugares que perciben como espacios de conflicto o de comportamientos ilícitos, y que pese a ello suelen compartir en forma cotidiana, los jóvenes muestran una actitud dual de rechazo-atracción. Los cuadros gris claro (Figura 2) señalan aquellos sectores donde los jóvenes evitan realizar sus trayectorias cotidianas, básicamente debido a conflictos y peleas protagonizadas por grupos rivales o por cualquier situación de disputa proveniente tanto de jóvenes como de cualquier habitante del sector.

Ahora, siguiendo con la simbología, los círculos representan lugares identificados por el alto consumo y tráfico de drogas en forma habitual, los cuales coinciden, precisamente, con las esquinas de dos poblaciones caracterizadas por la presencia de violencia o hechos ilícitos. Estos sucesos se presentan de día y de noche, por lo que los jóvenes consideran tales comportamientos como una conducta cotidiana que constituiría parte de las prácticas desarrolladas en estos lugares. Por último, el rectángulo negro en el mapa indica una calle reconocida como una de las vías que los adolescentes evitan debido a la posibilidad de enfrentarse con pandillas, lo que posibilitaría la ocurrencia de conflictos graves entre sus habitantes.

Mientras tanto, para el sector El Castillo, la Figura 3 muestra que las percepciones juveniles arrojan dos límites principales, la avenida Juanita (línea negra) y la calle Sexto de Línea (línea punteada), los cuales enmarcan dos subsectores a partir de los cuales se puede reconocer la pertenencia de los jóvenes a ciertos barrios, dependiendo del lado de la frontera en que se encuentren?.

9 Cabe señalar que, además de las vivencias propias de residir en estos lugares, las percepciones de los jóvenes se encuentran influenciadas por rumores o estigmas respecto de ciertas poblaciones o lugares, que indican que mientras más se penetra en el sector, más conflictivos suelen ser los comportamientos que allí se manifiestan. 
FIGURA 3 | Identificación de tipologías barriales según jóvenes. Sector El Castillo, La Pintana

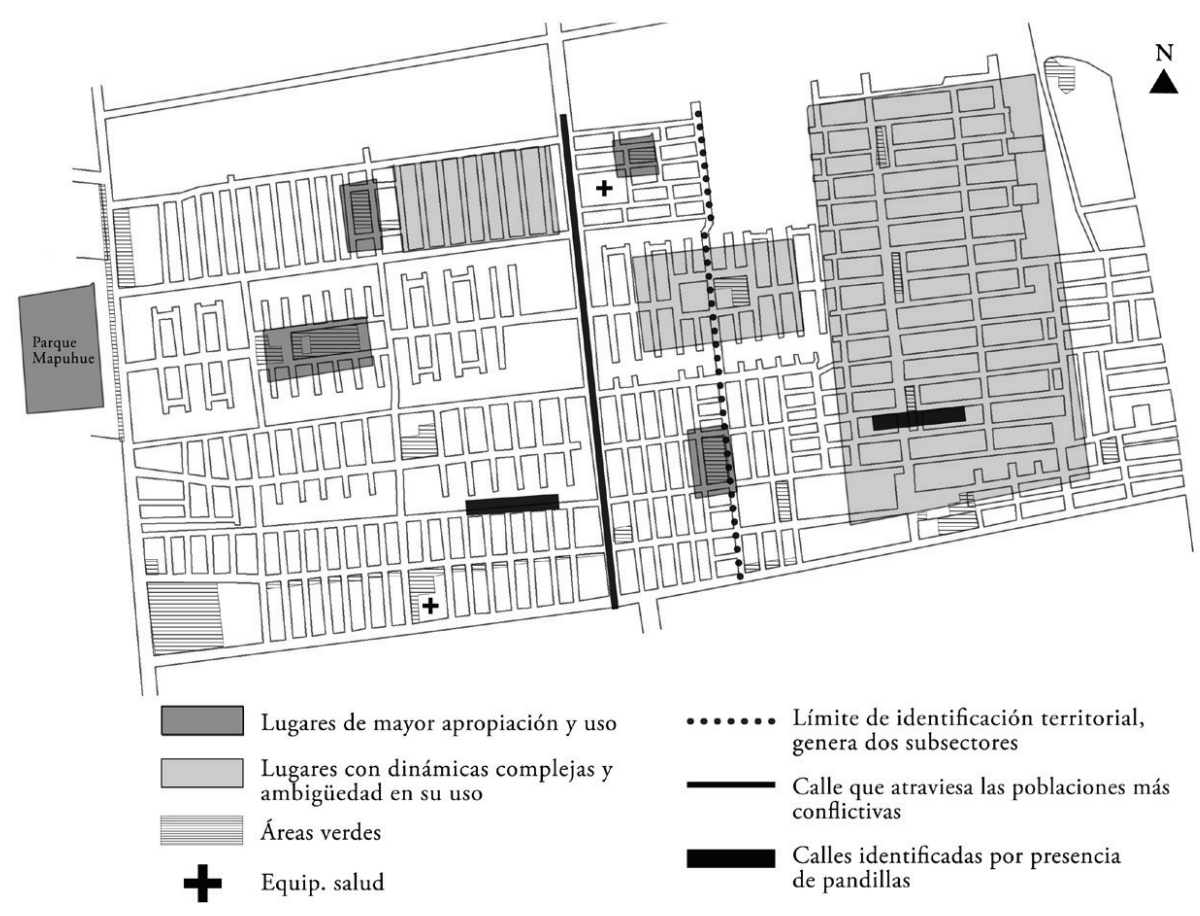

FUENTE ELABORACIÓN PROPIA A PARTIR DE INFORMACIÓN DE MAPAS PERCEPTUALES

En este sentido, estas dos fronteras reflejarían las situaciones de mayor conflicto o peligrosidad de sus calles, identificando un sector de mayor tranquilidad (poniente de la línea negra) y otro cargado de significaciones conflictivas o ilícitas (oriente de la línea negra). Asimismo, el segundo límite (línea punteada) indica mayor intensidad de este tipo de significados, orientando el nivel de conflictividad según la misma dirección que la anterior.

En El Castillo se aprecia mayor interés por utilizar ciertos espacios públicos por sobre otros; sin embargo, los jóvenes suelen identificar este sector como un espacio carente de lugares apropiados para distraerse y apropiarse de lo público. En efecto, indican sentirse parte de las plazas de su propia población, con la condición de que se encuentren cercanas a su hogar. Así, señalan como elemento relevante el compartir con su grupo de pares en estos lugares, como algo que otorga mayor valor al barrio, por lo que no necesitan recurrir a distancias lejanas para distraerse y compartir en grupo. Sin embargo, a pesar de esto, los jóvenes identifican graves carencias respecto de otras opciones para realizar sus actividades en grupo o individualmente.

Mientras tanto, respecto de los lugares de complejidad, en la Figura 3 los cuadros gris claro representan los espacios con dinámicas complejas en cuanto a la generación de situaciones violentas o la alta presencia de consumo y tráfico de drogas, así como la ocurrencia de hechos que se caracterizan por el uso de las armas. También se observan situaciones de conflicto cotidiano, como el consumo y tráfico de drogas, alta presencia de armas (balazos), peleas y enfrentamientos entre pandillas. Todos 
estos aspectos construyen una imagen del sector caracterizada como dual, según sean las trayectorias juveniles: mayor sensación de rechazo en aquellos que intentan alejarse de conductas ilícitas, y mayor atracción a los jóvenes que se integran a estos comportamientos. Aquellos que tratan de evitar este sector no reconocen abiertamente que lo hacen por temor; indican, más bien, que se trata de evitar problemas, o porque "es feo" o "es fome"; sin embargo, reconocen transitarlo de todas formas, para adquirir drogas.

Por otra parte, siguiendo con la Figura 3, existen dos calles representadas por rectángulos negros, donde los jóvenes señalan la existencia de conflictos entre los que comparten el territorio. Se trata, en particular, de rencillas desatadas por rivalidades entre grupos, los cuales posiblemente se constituyen como pandillas. Además, ambos sectores se ubican en lugares distintos de las fronteras señaladas por los jóvenes. Tal aspecto refuerza la presencia de dos subsectores al interior de El Castillo, como elemento que estimula a realizar investigaciones y nuevas propuestas orientadas a conocer posibles procesos de guetización y/o segregación en menor escala. Asimismo, ambos lados del límite focalizan los dos lugares de mayor presencia de jóvenes en conflicto con la justicia ${ }^{10}$.

$\mathrm{Al}$ reconocer la existencia de dos grandes dimensiones de abordaje de estas tipologías -estudio de los lugares de mayor apropiación, y de los lugares que presentan dinámicas complejas en cuanto a la presencia de hechos ilícitos-, entendemos que no estamos frente a diferencias mayores entre los sectores estudiados, y que fundamentalmente las dinámicas de apropiación del entorno barrial se sustentan en la predominancia de comportamientos violentos e ilícitos. Los jóvenes se involucran con distintos niveles de intensidad en tales comportamientos, lo que deriva en su posterior apreciación y uso de los espacios donde ellos tienen lugar.

En efecto, si bien es cierto que las prácticas complejas e ilícitas se encuentran diseminadas por gran parte del territorio estudiado, se pueden distinguir escenarios de mayor o menor participación de la población juvenil, tanto en términos de atracción o rechazo hacia estos lugares como en la elaboración mental respecto de sus vecindarios y, por ende, de sus vecinos. Es este un aspecto que entrega luces en torno a la relevancia del territorio en la generación y reafirmación de identidades juveniles, toda vez que contribuye a establecer su pertenencia a ciertos grupos y a diferenciarlos del resto.

\section{Visiones juveniles con signos de guetificación}

De acuerdo con los análisis de prácticas juveniles y de sus propias interpretaciones sobre las mismas, resulta significativa la búsqueda de normatividad, en el sentido de valorar los comportamientos de aquellas personas que se acercan de cierta forma a los valores y la moral predominante. No obstante esta valoración, los jóvenes sucumben en su accionar al imperativo territorial sustentado en las prácticas violentas o delictivas, que les permiten conformar un grupo y relacionarse en mayor profundidad a través de la identidad territorial.

10 Según los datos de la misma muestra mapeada por esta investigación. Sin embargo, se ha omitido su incorporación en el presente artículo. 
$\mathrm{H}:$... si antes yo entrenaba en una escuela de fútbol y no sé por qué nos fuimos de la escuela y ahí yo no tenía nada, no robaba, no fumaba marihuana tampoco, ni cigarro... (Juan, 15 años, El Roble).

E: Después, ¿̨por qué empezaste a hacer esas cosas?

H: No sé... es que el ambiente de aquí po', de la población... porque casi todos aquí fuman po', fuman cigarro o marihuana...

E: ¿Qué cosa fue lo que más te gustó de haber hecho o que hayan hecho con tus amigas, o algo que te haya gustado harto?

M: Sí, con otras cabras po', de repente... uuyyy... si de repente, cuando me las encontraba en las fiestas tenía que pelear con ellas. Una vez me querían pegar una puñalá y ahí yo tuve que quitarle la cuchilla y yo siempre tenía que andar con cuchilla, porque las locas igual andaban con cuchilla. Entonces siempre, era de todos los días andar con una cuchilla en los bolsillos, pa’ estar más segura... (Francisca, 17 años, El Roble).

Cabe señalar que estas conductas resultan ser prácticas "normales" dentro de las dinámicas grupales de estos jóvenes, contribuyendo así a generar una normalización de la conducta delictiva. Reflejan el peso de los atributos barriales en la construcción de identidad territorial, la cual se articula tanto desde el interior del grupo -según la percepción de pertenencia a una 'grupalidad' de forma instrumental-, como desde el exterior - de acuerdo con la imagen que buscan proyectar para su propio resguardo y para la apropiación y defensa del territorio al que pertenecen-.

Al experimentar esta apropiación del territorio, un hecho característico de estos barrios es el reconocimiento de los habitantes entre sí como una estructura de control de sus acciones. Este mecanismo, junto a la configuración identitaria y la permanencia de los jóvenes desde su nacimiento en estos barrios, redunda en un nulo desplazamiento de ellos hacia otros sectores de la comuna y, en menor medida aún, hacia otras comunas de Santiago. Tal como manifiesta un joven de El Castillo:

E: Y pa' otros lugares fuera de La Pintana, pa' otros lugares de Santiago, pa'l centro... ¿ ¿vas?

H: No, no voy... Si paso en el puro Castillo metío... si pasamos puro aquí...

(Diego, 16 años, El Castillo).

Entre las prácticas grupales adquiridas por los jóvenes, las experiencias colectivas, con toda su intensidad, ocupan un nivel de centralidad mayor que los vínculos profundos de amistad al interior del grupo de pares. Entre dichas experiencias se encuentran muy arraigadas las actividades delictivas y violentas que llevan a cabo en grupo, así como el consumo de marihuana y alcohol. Los jóvenes les otorgan gran relevancia a las oportunidades en que han incurrido en robos, en "quitás de drogas" o "mexicanas", cuando han participado en peleas y otras actividades de esta índole.

En efecto, los grupos y el entorno proveen al joven de elementos culturales y materiales de realización y aprendizaje en su trayectoria, los mismos que no logra encontrar en la familia ni en la escuela. Existe, así, una función propia, "depositaria de estos grupos, como el acceder a identidad con un grupo de pertenencia a un territorio propio (...) el joven accede a roles y a formas de prestigio social alternativos, e incluso un sentido de vida que la sociedad le niega" (Cooper, 2005, p. 109). 
De esta forma, la socialización entre grupos de pares se materializa a través de una profunda tensión entre la búsqueda de los jóvenes por sentirse parte de la sociedad y los medios que utilizan para realizar esta integración -tensión que podríamos denominar de desintegración global e integración local-.

En el sentido descrito, existe una suerte de "culturicidad de lo barrial" (Gravano, 2005): al identificar pautas conductuales e imágenes en torno al vecindario que se sustentan como valores en sí mismos, el barrio surge como espacio que plantea la posibilidad de constituirse en cultura (subcultura, de acuerdo con Cooper) o mundos propios que se desarrollan paralelamente a la cultura dominante. Desde esta perspectiva existen ciertas señales de guetización de los barrios de La Pintana. Vale decir, los hallazgos del estudio se vinculan con una característica del gueto, la cual radica en que la relación entre el grupo que ostenta la característica de gueto y el resto de la ciudad, es una relación marcada por una separación que no siempre es categórica ni visible, sino que "se presenta de forma aparente, es una separación de 'mundos vividos', no de 'sistemas', para usar una distinción conceptual elaborada por Habermas (1964). Esa distinción se refiere a las experiencias y relaciones concretas de sus ocupantes, no a los lazos subyacentes que los anclan con firmeza al conjunto metropolitano, si bien en la modalidad de la exclusión" (Wacquant, 2001, p. 39). En esta línea, se aprecia en los intereses o expectativas de los jóvenes que ellos se proyectan más alejados de conductas delictivas que permaneciendo en ellas, situación donde lo primordial radica en valores compartidos por el resto de la sociedad, como el trabajo, la familia, la tranquilidad, el bienestar económico y, ciertamente, todo eso fuera del barrio:

E: ¿Hay algo que te gustaría hacer en tu vida?

H: Irme de aquí me gustaría a mí... Me gustaría irme pa’ una parte tranquila

(Carolina, 15 años, El Castillo).

E: ¿Qué es importante para ti en la vida?

H: A mí me gustaría tener mi familia como todos, y ahí tener su casita y nada más y trabajar tranquilo; si ya era, ya era el cabro chico... (Felipe, 17 ańos, El Castillo).

E: ¿Y qué te gustaría ser en tu vida?

H: Ser empresario... de un tipo así que tuviera un negocio gigante, así una ferretería, algo así po', unas cinco ferreterías... y ponerles nombre.

E: ¿Por qué te gustaría eso?

H: ¿`Ser empresario? Porque me gusta manejar plata (Ismael, 15 años, El Castillo).

Los jóvenes revelan sentir la necesidad de involucrarse en los patrones sociales y culturales externos predominantes, y ven en el consumo la posibilidad de inclusión en los estilos de vida del resto de la ciudad. En este sentido, "la identidad unificada [del gueto] no puede evitar llevar la marca de la ambivalencia (...) tiene, por lo tanto, una tendencia a fomentar entre sus miembros sentimientos de duda y odio de sí mismos, disimulación del origen 'pasando (por otro)', la perniciosa derogación de la propia clase, e incluso una fantasiosa identificación con los dominantes" (Wacquant, 2001, p. 79).

Desde esta perspectiva, los barrios analizados presentan algunas características de guetización, en el sentido de constituirse en áreas urbanas con estigma, restricción y confinamiento espacial. Sin embargo, observamos que el concepto de gueto no da 
cuenta de todos los elementos existentes en el contexto empírico examinado. Más bien, respecto de la existencia de instituciones específicas a estos grupos, encontramos que -en términos generales- los habitantes de la comuna mantienen lazos permanentes con el resto de la urbe, proporcionando mano de obra y accediendo a algunos servicios básicos. Ello no obstante la fuerte presencia de empleos informales (e ilegales) en los sectores estudiados, como formas alternativas de obtener ingresos frente a los altos índices de desempleo, o dadas las menores oportunidades de capacitación y formación especializada para los segmentos que allí residen.

\section{Conclusiones}

Con el fin de comprender las dinámicas desarrolladas en barrios segregados de la ciudad de Santiago, particularmente de jóvenes con compromiso delictual, se ha indagado en dimensiones que caracterizan los estilos de vida compartidos en sus vecindarios y la forma en que estos son articulados por los mecanismos de socialización -institucionalizados o no-, que finalmente orientan el actuar de los habitantes pobres de áreas periféricas de la urbe.

Territorialidad, formación identitaria juvenil y socialización en barrios segregados La territorialidad desempeńa un rol fundamental para los jóvenes. Sus grupos de pares están conformados por adolescentes que se acompańan en un proceso de crecimiento e inserción social, proceso generalmente caracterizado por poseer mayores significados de territorialidad que de amistad: los lazos entre pares son instrumentales, a modo de una racionalidad con arreglo a fines, como identificarse, blindarse, estructurar sentido y protección a sus vidas en resguardo del entorno en que viven.

Se concluye que el grupo de pares opera como mecanismo de socialización a través de tres ejes fundamentales: la función de cohesión para el grupo que cumple el consumo de drogas y alcohol ${ }^{11}$; los enfrentamientos entre grupos rivales como forma de apropiación y defensa del territorio; y el delito como actividad constitutiva y sentido de pertenencia al grupo, además de ser una forma de integración local.

Por su parte, el espacio público del barrio constituye un mecanismo fundamental de socialización para estos jóvenes, el cual se define a partir de dos elementos: el que las personas sean reconocidas por el resto, y la inseguridad percibida que define al barrio. La relación entre el espacio público y las conductas violentas/delictivas se articula en torno a cuatro ejes: el peso de los atributos barriales en la conformación del grupo de pares; el uso del espacio y la movilidad limitados por la segregación; la naturalización de la violencia en estos espacios; y la relación barrio-drogas en todas sus facetas (el consumo problemático, el uso de armas para su venta, la dependencia económica de estos barrios respecto del microtráfico, los conflictos familiares, etcétera).

11 Tal como arrojan algunos estudios que otorgan un importante papel al vínculo entre violencia y droga. Para esto se puede revisar Otero (2006) y Redondo y Garrido (2004.) 
FIgura 4 | Población El Castillo

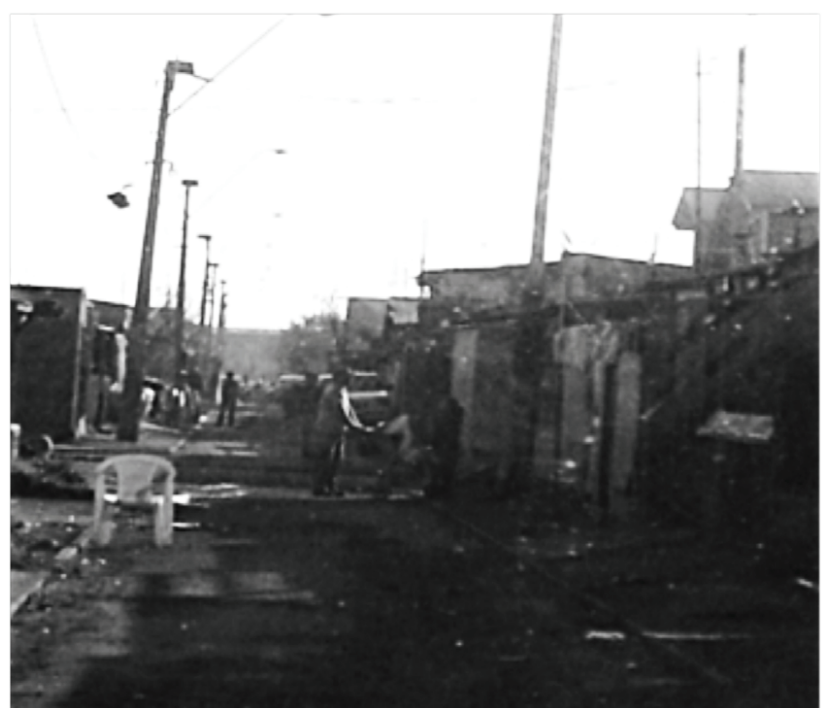

La socialización para los jóvenes se realiza a partir de una relación de interdependencia que estructura (y es estructurada en) las experiencias de los jóvenes pobres urbanos, donde el barrio se superpone al peso de los grupos de pares para construir las trayectorias juveniles en su interiorización de prácticas delictivas. En esta relación, la familia y la escuela constituyen los mecanismos mediante los cuales se internaliza y reproduce el marco normativo de dichas prácticas.

En definitiva, el delito es una forma de integración social a escala local, puesto que las relaciones interpersonales, la definición de identidad personal y colectiva, las actividades y su desplazamiento -dentro y fuera del barrio- y las proyecciones de vida, entre otras cosas, adquieren un sentido de territorialidad en estos sectores. Pero este sentido no es neutro, sino que se entrelaza con procesos de definición hacia dentro y fuera del espacio estudiado, en virtud de la permanente tensión con las identidades que se generan en otros territorios de la comuna y de la ciudad.

figura 5 | Población El Castillo

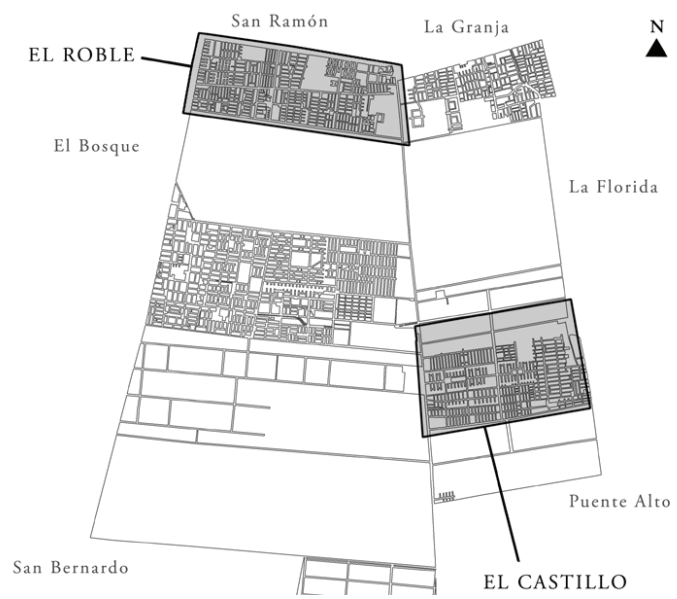


Hacia una propuesta de estudio: síntomas de guetización en La Pintana

Es posible sostener que el territorio y su "culturicidad" -su posibilidad de constituirse en cultura, entendida como sistema de representaciones y prácticas compartidas socialmente en torno a valores distintivos (Gravano, 2005)- operan como elementos estructurantes de relaciones violentas, en el sentido de que diversos conflictos entre personas o grupos se resuelven a través de esta forma. Las maneras de socialización "peligrosas" percibidas por los jóvenes, se dan en un contexto de significaciones que atribuyen gran naturalidad a la violencia como método de resolución de conflictos. Asimismo, gran parte de las relaciones familiares y territoriales logran reproducirse a sí mismas en este confinamiento espacial. Tal como señala Wacquant (2001), el gueto vendría a constituir un "instrumento sociorganizacional que emplea el espacio para reconciliar dos propósitos antinómicos: maximizar las ganancias materiales extraídas de un grupo considerado mancillado y mancillante, y minimizar el contacto íntimo con sus miembros para alejar la amenaza de la corrosión y el contagio simbólicos que llevan consigo" (p. 75).

Lo anterior se comprende a partir de la historia de la comuna de La Pintana, así como de otras de la capital, cuyo origen se encuentra en las erradicaciones y radicaciones forzadas ocurridas en el contexto de la dictadura militar en nuestro país. Tales procesos constituyeron un hito fundante encarnado en violencia social, a través de la cual se espacializó la exclusión experimentada por diversos grupos en otros ámbitos (laboral, educacional, cultural, etcétera). Por esta razón, los síntomas de guetización encontrados en los espacios urbanos así constituidos se orientan -en palabras de Wacquant (2001) - hacia una agudización de la frontera "entre la categoría excluida y la población que la rodea"; y lo hacen, continúa el autor, "al profundizar el abismo sociocultural entre ellas: hace a sus residentes objetiva y subjetivamente más disimilares de otros habitantes de la ciudad sometiéndolos a condicionamientos especiales, de manera que los patrones de cognición y conducta a los que dan lugar tienen todas las posibilidades de ser percibidos desde afuera como singulares, exóticos, incluso aberrantes...” (p. 79). Desde este punto de vista, el gueto sería una variable susceptible de ser analizada en poblaciones con las características detectadas en los dos sectores en estudio, ambos con presencia de elementos propiciadores -geográfica y culturalmente- de aprendizajes de comportamientos delictivos.

Finalmente, se plantean cuestionamientos en torno a la idea de orden social y normatividad, debido al predominio en estos conceptos de las perspectivas de lo positivo, lo fecundo, lo estable. Pero visto desde la particularidad empírica, el (des) orden de estos sectores podría ser un orden social con posibilidades de participación para sus habitantes, puesto que se reproduce, se mantiene en el tiempo y representa perspectivas de desarrollo para las personas que lo habitan. Esto se refuerza en la medida en que es posible interrogarse sobre lo siguiente: si no existiera este desarrollo económico informal e ilegal, ni las especificidades cognitivas y conductuales en torno a la sobrevivencia, basada en la constante defensa respecto del otro y la identificación territorial de estos grupos, ¡sobre qué elementos se sustentarían estos grupos y sus prácticas en el contexto de exclusión permanente que atraviesa gran parte de la población? 
Desde la cultura hegemónica, estos jóvenes son lo feo, lo malo, el desorden, un mundo que no encaja en los patrones culturales predominantes. Por eso están en la periferia. Pero ellos generan un nuevo orden para sus vidas, realizando algo parecido a una integración hacia dentro, a partir de lo que pueden hacer y a lo que pueden acceder. Y por esa vía construyen una subcultura que no le disputa su lugar a la cultura dominante, pero que provee a sus habitantes de códigos, normas, rutinas y dinámicas, a través de los cuales se percibe que es particular, que no es transversal al resto de la sociedad, pero que tampoco intenta competir con esta normatividad. Se trata de prácticas que se realizan en función de un territorio -consciente o inconscientemente- marginal.

\section{Referencias bibliográficas}

Arriagada C. \& Simioni, D. (2000) Acceso al suelo, impuestos locales y financiamiento del desarrollo urbano: el caso de Santiago de Chile. Cambridge, MA: Lincoln Institute Research Report. Recuperado del sitio Web del Lincoln Institute Research: http:// www.lincolninst.edu/pubs/708_ACCESO-AL-SUELO--IMPUESTOSLOCALES-YFINANCIAMIENTO-DEL-DESARROLLO-URBANO

Arriagada, I. \& Godoy, L. (2000). Prevenir o reprimir: Falso dilema de la seguridad ciudadana. Revista de la CEPAL, 70, pp. 107-131. Recuperado de http://www.eclac.org/ publicaciones/xml/4/19264/arriagada.pdf

Arriagada, C. \& Rodríguez, J. (2004). Segregación residencial en la ciudad latinoamericana. Revista EURE, 30(89), 5-24. http://dx.doi.org/10.4067/S0250-71612004008900001

Balbo, M., Jordán, R. \& Simioni D. (Comps.). (2003). La ciudad inclusiva. [Versión electrónica] Cuadernos de la Cepal, 88. Santiago: Cepal. Recuperado de http://www.cepal.org/ publicaciones/xml/7/14237/lcg2210p.pdf

Contreras, P. \& Weason, M. (2006). Trabajo adolescente y comportamientos en conflicto con la ley: socialización en el espacio laboral. En Pontificia Universidad Católica de Chile \& Paz Ciudadana, Segundo Simposio Nacional de Investigación sobre Violencia y Delincuencia. Santiago: Creativas S.A.

Cooper, D. (2002). Criminología y delincuencia femenina en Chile. Santiago: LOM Ediciones. Cooper, D. (2005). Delincuencia y desviación Juvenil. Santiago: LOM Ediciones.

Dammert, L. (2005). Violencia criminal y seguridad ciudadana en Chile. Serie Políticas Públicas, 109. Santiago de Chile: CEPAL. Recuperado de http://www.eclac.org/publicaciones/ xml/1/22061/sps_109.pdf

Dammert, L. \& Oviedo, E. (2004). Santiago: Delitos y violencia urbana en una ciudad segregada. En C. De Mattos et. al. (Eds.), Santiago en la globalización: ¿Una nueva ciudad? Santiago: Ediciones SUR y EURE.

De Mattos, C. (1999). Santiago de Chile, Globalización y expansión metropolitana: lo que existía sigue existiendo. EURE, 25(76), 29-56. http://dx.doi.org/10.4067/S025071611999007600002 
De Mattos, C. (2002). Transformación de las ciudades latinoamericanas, ¿impactos de la globalización? EURE, 28(85), 5-10. http://dx.doi.org/10.4067/S025071612002008500001

De Mattos, C. (2004). Santiago de Chile, ¿ejemplo de una reestructuración capitalista global? EURE, 30(91), 111-120. http://dx.doi.org/10.4067/S0250-71612004009100002

De la Puente, P., Torres, E., Muñoz, P., Sepúlveda, R. \& Arditi, C. (1993). Familia, vecindario y comunidad: un modelo sistémico para la interpretación del desarrollo progresivo. Estudios Sociales, 76(trimestre 2), 149-167.

Ducci, M. E. (2004). Las batallas urbanas de principios del tercer milenio. En C. De Mattos et al. (Eds.), Santiago en la globalización: ¿una nueva ciudad? Santiago: Ediciones SUR / EURE Libros.

Fernández, J. (2004) Delincuencia y exclusión social: Estructuras sociales y procesos de socialización imbricados. En Pontificia Universidad Católica de Chile y Paz Ciudadana, Primer Simposio Nacional de Investigación sobre Violencia y Delincuencia (pp. 33-47). Santiago: Creativas S.A.

Frühling, H. \& Sandoval, L. (1997). Percepciones de inseguridad y realidad delictual en tres comunas populares de Santiago. Estudios Públicos, 68(primavera). Recuperado de http://bit.ly/1ezSvty.

García-Pablos A. (1994). Criminología. Una introducción a sus fundamentos teóricos para Juristas. Valencia: Tirant lo Blanc.

Gravano, A. (2005). El barrio en la teoría social. Buenos Aires: Espacio Editorial.

Hidalgo, R. (2007). ¿'Se acabó el suelo en la gran ciudad? Las nuevas periferias metropolitanas de la vivienda social en Santiago de Chile. EURE, 33(98), pp. 57-75. http://dx.doi. org/10.4067/S0250-71612007000100004

Kaztman, R. (2001). Seducidos y abandonados: el aislamiento social de los pobres urbanos, Revista de la CEPAL, 75, 171-189. Recuperado de http://www.eclac.org/publicaciones/ xml/6/19326/Katzman.pdf

Kaztman, R., Corbo, G., Filgueira, F., Furtado, M., Gelber, D., Retamoso, A. \& Rodríguez, F. (2003). La ciudad fragmentada: Mercado, territorio y delincuencia en Montevideo. Documentos de trabajo, Project Latin American Urbanization in the Late 20th Century: A Comparative Study, No 2. Montevideo. Recuperado de http://www.redligare.org/ IMG/pdf/montevideo_ciudad_fragmentada.pdf

Lunecke, A. (2004). Violencia en los barrios estigmatizados, un desafío de sociedad. Mensaje (Santiago), 553 (octubre).

Lunecke, A. \& Ruiz, J. (2006). Barrios urbanos críticos en materia de violencia y delincuencia. En Pontificia Universidad Católica de Chile y Paz Ciudadana, Segundo Simposio Nacional de Investigación sobre Violencia y Delincuencia. Santiago: Creativas S.A.

Mettifogo, D. \& Sepúlveda, R. (2005). Trayectorias de vida de jóvenes infractores de ley. Santiago: Centro de Estudios de Seguridad Ciudadana (CESC), Universidad de Chile.

Municipalidad de La Pintana. Secretaría de Planificación Comunal (2007). Guía de Información Comunal. Santiago, Chile.

Programa de las Naciones Unidas para el Desarrollo (pNUd). (1998). Desarrollo Humano en Chile, las paradojas de la modernización. Santiago: Autor.

Redondo, S. \& Garrido, V. (2004). Violencia y delincuencia juvenil. Mendoza: Ediciones Jurídicas Cuyo. 
Rodríguez, A. (2005). Vivienda social / Violencia intrafamiliar: Una relación inquietante. Boletín Temas Sociales 53 (Santiago: SUR Corporación de Estudios Sociales y Educación). Recuperado de http://www.sitiosur.cl/r.php?id=151

Rodríguez, A. \& Sugranyes, A. (2004). El problema de vivienda de los "con techo". EURE, 30(91), 53-65. http://dx.doi.org/10.4067/S0250-71612004009100004

Rodríguez, A. \& Winchester, L. (2001). Santiago de Chile. Metropolización, globalización, desigualdad. Revista EURE, 27(80), 121-139. http://dx.doi.org/10.4067/S025071612001008000006

Sabatini, F. (1999). La segregación espacial y sus efectos sobre los pobres y la seguridad ciudadana. En Ministerio de Vivienda y Urbanismo, Espacio Urbano, Vivienda y Seguridad Ciudadana. Relatoría de Seminario homónimo. Santiago, Chile.

Sabatini, F. (2000). Reforma de los mercados de suelo en Santiago, Chile: efectos sobre los precios de la tierra y la segregación residencial. EURE, 26(77), 49-80. http://dx.doi. org/10.4067/S0250-71612000007700003

Saraví, G. (2004). Segregación urbana y espacio público: los jóvenes en enclaves de pobreza estructural. Revista de la CEPAL, 83, 33-48. Recuperado de http://www.eclac.cl/ publicaciones/xml/8/19418/lcg2231eSaravi.pdf

Tironi, E., Weinstein, E. \& Martínez, J. (1990). Personas y escenarios en la violencia colectiva. Vol. II de La violencia en Chile. Santiago: Ediciones SUR.

Tijoux, M. E. (1995). Jóvenes pobres en Chile: nadando en la modernidad y la exclusión. Última Década, 3, 1-9. Recuperado de http://www.redalyc.org/articulo.oa?id=19500303

Wacquant, L. (2001). Parias urbanos. Marginalidad en la ciudad a comienzos del milenio. Buenos Aires: Ediciones Manantial. 